\title{
Caracterización del profesorado con perfil investigador en universidades colombianas ${ }^{1}$
}

\author{
Characterization of the Professors with a Research Profile at Colombian Universities ${ }^{2}$ \\ Caracterização do corpo docente com perfil de pesquisa nas universidades colombianas ${ }^{3}$
}

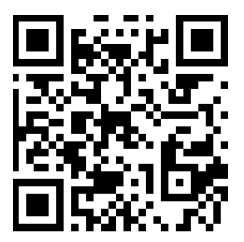

Salim Chalela-Naffah

Universidad Autónoma de Barcelona

Barcelona, España

salim.chalela@e-campus.uab.cat

https://orcid.org/0000-0003-3982-2696

David Rodríguez-Gómez

Universidad Autónoma de Barcelona

Barcelona, España

david.rodriguez.gomez@uab.cat

http://orcid.org/0000-0001-9845-0744

Recibido • Received • Recebido: 04 / 03 / 2019

Corregido • Revised • Revisado: 08 / 05 / 2020

Aceptado•Accepted•Aprovado: 03 / 08 / 2020

\begin{abstract}
Resumen: Las dinámicas de producción de conocimiento, derivadas de la actividad científica realizada en las instituciones de educación superior, han sido un fenómeno ampliamente estudiado desde mediados del siglo XX. En Colombia, el análisis de las capacidades que desarrollan las universidades para la investigación ha cobrado importancia en los últimos treinta años como consecuencia de la intención de fortalecer su sistema nacional de ciencia, tecnología e innovación, liderado por el Ministerio de Ciencia, Tecnología e Innovación. Este trabajo presenta una caracterización del profesorado con perfil investigador (condiciones institucionales y factores individuales) vinculados a universidades colombianas. Mediante un estudio cuantitativo, no experimental, desarrollado en 2018, se aplicó un cuestionario autoadministrado a una muestra representativa de 379 docentes con perfil investigador, líderes de grupos de investigación, adscritos a diferentes universidades del país. El análisis de datos se realizó por medio de pruebas no paramétricas $H$ Kruskal Wallis y U de Mann Whitney. Se encontró que algunas variables como el área disciplinar de formación del profesorado, el tipo de vinculación laboral a la universidad y las concepciones de la investigación que tiene están asociadas al tipo de crecimiento de la productividad científica que muestran las universidades.
\end{abstract}

${ }^{1}$ El artículo es resultado del proyecto de investigación doctoral: La gestión de la investigación en universidades colombianas. Doctorado en educación de la Universidad Autónoma de Barcelona.

${ }^{2}$ The paper resulted from a doctoral research project titled: Research Management in Colombia's Universities. PhD program in Education. Autonomous University of Barcelona.

${ }^{3} \mathrm{O}$ artigo é resultado do projeto de pesquisa de doutorado: a gestão da pesquisa nas universidades colombianas. Doutorado em educação pela Universidade Autônoma de Barcelona. 
http://doi.org/10.15359/ree.24-3.17

http://www.una.ac.cr/educare

educare@una.ac.cr

En este sentido, el estudio aporta evidencia empírica, tanto en las condiciones institucionales (estructura organizacional para la investigación y prácticas de gestión de la investigación) como en factores individuales, que explica la existencia de las diferencias en el desarrollo y consolidación de las dinámicas de producción científica en las universidades colombianas.

Palabras claves: Educación superior; profesorado-investigador; productividad; ambiente institucional; universidades.

\begin{abstract}
The knowledge production dynamics, resulting from scientific activities in higher education, have been a phenomenon widely studied in the last decades. In Colombia, the scientific research capabilities in higher education institutions have been the focus of attention in the last thirty years thanks to the strengthening of the national system of science, technology and innovation, at the head of the Ministry of Science, Technology and Innovation. This paper presents an analysis of the characteristics of professors with a research profile at Colombian universities. Through a quantitative, no experimental examination in 2018, a questionnaire was applied to 379 teachers, who are research group leaders and are working in Colombian universities. The data analysis was conducted using non-parametric tests $\mathrm{H}$ Kruskal Wallis and $\mathrm{U}$ of Mann Whitney. As a result, some variables such as the professor's field of knowledge, kind of university contract, and research conceptions of professors are related to the kind of scientific productivity growth in the universities. In this way, the study contributes to understanding how the institutional environment (organizational structure and management practices) and the individual's factors explain the differences over the development and consolidation of research activities in Colombian universities.
\end{abstract}

Keywords: Higher Education; teacher-researchers; productivity; institutional environment; universities.

Resumo: A dinâmica da produção de conhecimento, derivada da atividade científica realizada em instituições de ensino superior, tem sido um fenômeno amplamente estudado desde meados do século XX. Na Colômbia, a análise das capacidades que as universidades desenvolvem para pesquisa ganhou importância nos últimos trinta anos, como consequência da intenção de fortalecer seu sistema nacional de ciência, tecnologia e inovação, liderado pelo Ministério da ciência, tecnologia e inovação. Este artigo apresenta a análise das características do corpo docente com perfil de pesquisa nas universidades colombianas. Através de um estudo quantitativo não-experimental, desenvolvido em 2018, aplicou-se um questionário auto administrado a uma amostra representativa de 379 professores com perfil de pesquisa, líderes de grupos de pesquisa, pertencentes a diferentes universidades do país. A análise dos dados foi realizada por meio dos testes não paramétricos de H Kruskal Wallis e $U$ de Mann Whitney. Constatou-se que algumas variáveis, como a área disciplinar da formação de professores, o tipo de trabalho vinculado à universidade e as concepções de pesquisa que estas têm estão associados ao tipo de crescimento da produtividade científica das universidades. Neste sentido, o estudo fornece evidência empírica, relacionada tanto com as condições institucionais (estrutura organizacional para a pesquisa e práticas de gestão de pesquisa) e fatores individuais, o que explica a existência de diferenças no desenvolvimento e consolidação das atividades de pesquisa nas universidades colombianas.

Palavras-chave: Ensino superior; professor-pesquisador; produtividade; ambiente institucional; universidades. 


\section{Introducción}

Las dinámicas de producción de conocimiento, derivadas de la actividad científica realizada en las instituciones de educación superior como motor de desarrollo de las sociedades han sido un fenómeno ampliamente estudiado desde mediados del siglo XX (Altbach y Wit, 2019). Organizaciones públicas y privadas, nacionales e internacionales cuantifican el impacto de los resultados de investigación y evalúan la productividad científica de las universidades con la generación de métricas y ránquines que han obligado a replantear elementos clave de los sistemas de educación superior, así como de las políticas de ciencia y tecnología en la mayoría de los países (Frenken et al., 2017; Gralka et al., 2019).

La eficiencia y productividad científica de las investigaciones en revistas de alto impacto (Daraio, 2019), el número de citaciones de artículos y libros publicados (Bornmann y Leydesdorff, 2017), los tipos de colaboraciones internacional (proyectos conjuntos, coautorías) (Macfarlane, 2017), la clasificación de grupos y centros de investigación (Kyvik y Aksnes, 2015), la cantidad de recursos nacionales e internacionales gestionados para financiar proyectos (Cattaneo et al., 2016), el nivel de formación de los grupos investigadores y las disciplinas en las que se produce el conocimiento (Abramo et al., 2017), la reputación individual y los salarios (Kwiek., 2018), la satisfacción laboral al interior de las instituciones (Albert et al., 2018), entre otras, son algunas de las variables que han sido estudiadas a lo largo del tiempo como factores asociados a la productividad científica de las universidades.

En Colombia, el análisis de la productividad científica de las universidades ha cobrado importancia en los últimos treinta años como consecuencia de la intención de fortalecer su sistema nacional de ciencia, tecnología e innovación. Desde esta perspectiva, el gobierno nacional otorgó a Colciencias, hoy Ministerio de Ciencia, Tecnología e Innovación, como entidad rectora de la política nacional de ciencia, tecnología e innovación, la facultad de generar estrategias de fomento a las actividades científicas que se desarrollan en el país como becas de formación de talento humano, financiación de programas y proyectos de investigación; así como directrices para el reconocimiento y la evaluación del desempeño de centros, grupos de investigación e personal investigador (Plata, 2013). La acción de Colciencias ha dejado como resultado un aumento cuantitativo de las capacidades científicas nacionales en los últimos treinta años, y han evidenciado entre otras, en (1) un mayor número de docentes con título de doctorado; (2) la apertura de programas de formación doctoral en el país; (3) el aumento en el número de grupos de investigación e y sujetos investigadores adscritos a universidades, organizaciones públicas y privadas; (4) el incremento en las publicaciones en revistas de alto impacto; y (5) una mayor inversión de recursos institucionales (universidades) para el desarrollo de actividades de ciencia y tecnología (Ospina Bozzi et al., 2016).

Este aumento en las capacidades científicas nacionales ha sido examinado en algunas investigaciones que han analizado las dinámicas de evaluación científicas de la política nacional de 
http://doi.org/10.15359/ree.24-3.17

http://www.una.ac.cr/educare

educare@una.ac.cr

ciencia, tecnología e innovación en Colombia (Lucio et al., 2017), el crecimiento de la productividad científica de las universidades (Bucheli et al., 2012), el efecto de los incentivos económicos en la producción académica del profesorado (Méndez Sayago y Vera Azaf, 2015), el análisis de sistemas dinámicos para identificar capacidades de investigación universitaria (Delgado Hurtado et al., 2017), entre otras. Sin embargo, en el país aún no existen suficientes estudios, desde el ámbito de la investigación educativa, que ahonden en la caracterización de las condiciones sociodemográficas, institucionales y los factores individuales que describan al profesorado con perfil investigador en las universidades, lo cual se convierte en el objetivo de este trabajo.

\section{Revisión bibliográfica}

A lo largo de la historia, las universidades se han caracterizado por desplegar, en un mayor o menor grado, actividades de investigación como aporte al desarrollo social y económico, lo que ha generado la necesidad de dotarse de condiciones, institucionales e individuales, suficientes que les permitan generar conocimiento pertinente en un mundo globalizado y cada vez más competitivo (Upadhyaya y Rajasekharan, 2019).

Al hacer referencia a condiciones institucionales para la investigación universitaria, Nguyen y Meek (2016) analizaron la existencia de políticas, directrices y recursos institucionales como estrategias de gestión esenciales para generar una cultura organizacional que propicie el desarrollo de la investigación en el seno de la universidad. Ion y Castro-Ceacero (2017) identificaron que la infraestructura física, el tamaño de las facultades, centros y departamentos, los procesos de formación del personal investigador, la especificidad de plazas para el desarrollo de la actividad investigativa y el ambiente laboral, son clave para que se configure una cultura de la investigación en la universidad. Edgar y Geare, (2013) analizaron variables como la asignación de tiempo en el plan de trabajo para el desarrollo de procesos de investigación, la disponibilidad de recursos financieros, técnicos y humanos, el tipo de vinculación laboral y el grado de libertad para desarrollar actividades científicas han sido estudiadas como condiciones institucionales que determinan al profesorado para desarrollar investigación en instituciones de educación superior. Albert et al., (2018) han explorado en la satisfacción laboral del profesorado y Amara et al. (2015); Méndez Sayago y Vera Azaf (2015) estudiaron el otorgamiento de incentivos, financieros y no financieros, por productividad científica.

En cuanto a los factores individuales, Brew (2001) y Brew et al. (2016) propusieron cuatro concepciones diferentes de lo que el profesorado entiende por investigación en la educación superior: (1) la concepción dominó, en la que la investigación se define como un proceso de tareas, eventos cosas, actividades, problemas, técnicas experimentos, asuntos, ideas o preguntas separadas, cada una de las cuales es presentada como distintiva; (2) la concepción de capas que define la investigación como proceso de búsqueda de respuestas a partir de la generación de 
http://doi.org/10.15359/ree.24-3.17

preguntas generales; (3) la concepción de intercambio, en la que el énfasis de la investigación está puesto en los resultados y cómo estos se convierten en prestigio personal para que el personal investigador pueda introducirse en otras comunidades (lógica comercial); y (4) la concepción de travesía personal entendiendo la investigación como un aprendizaje y un instrumento para la transformación del individuo como sujeto social (Brew, 2001; Brew et al., 2016).

Stubb et al. (2014) compartieron las tipologías mencionadas en el párrafo anterior y partiendo de estas dieron cuenta de que la investigación universitaria responde a concepciones centradas en los procesos y concepciones focalizadas en los productos. Concluyeron que la investigación que pone su mirada en los productos tiene como finalidad satisfacer requerimientos académicos e individuales de los equipos de investigación; mientras que las concepciones centradas en los procesos apuntan a la generación de conocimiento y el aporte social de la investigación para el avance de la ciencia (Stubb et al., 2014).

El grado de colaboración y compañerismo es otra variable fundamental que ha sido estudiada, dado que permite al profesorado universitario generar procesos de investigación e intercambio con sus pares de la universidad o con colegas nacionales o internacionales. Macfarlane (2017) disertó sobre diferentes concepciones respecto a la colaboración científica, donde consideró relaciones entre universidades, universidades e industrias y centros de investigación e personas investigadoras fundamentadas en el ideal de generar resultados de investigación conjuntos que amplíen el avance de la ciencia en temas comunes y la innovación. Este trabajo en red va acompañado de la creciente necesidad de los sujetos investigadores de trabajar en colaboración, lo cual se ve evidenciado en el crecimiento del número de resultados de investigación desarrollados en coautoría, así como en la participación de docentes en eventos internacionales afines a sus áreas de conocimiento (Abramo et al., 2017).

El tipo de producción científica es otra variable que se relaciona con el perfil investigador del profesorado. La preferencia por generar resultados de manera individual o en coautoría (Frenken et al., 2017), las bases de datos en las que se encuentran indexadas las revistas y la preferencia por publicar artículos o libros como resultados de investigación pueden caracterizarlos (Abramo y D’Angelo, 2014).

Adicionalmente, se ha encontrado que características sociodemográficas como el sexo, la edad, la región en la que se adelantaron los estudios doctorales (Amara et al., 2015) y la disciplina en la que se desarrolla investigación tipifican el perfil de desempeño investigador del profesorado universitario (Daraio, 2019).

Por otro lado, la productividad científica de las universidades se define como el proceso de generación de conocimiento en forma de publicaciones de los trabajos de investigación (ie., artículos publicados en revistas, libros, capítulos de libros, ponencias en conferencias, documentos de trabajo) o generación de innovaciones científicas (ej. patentes, modelos de 
http://doi.org/10.15359/ree.24-3.17

http://www.una.ac.cr/educare

educare@una.ac.cr

utilidad o desarrollos tecnológicos). Partiendo de esta consideración, algunos aportes al estudio de la productividad científica de las universidades se han generado desde la cienciometría que evalúa aspectos como el crecimiento cuantitativo de la ciencia, la estructura de comunicación entre las personas científicas, el impacto y la calidad de las publicaciones, entre otras variables (Bornmann y Leydesdorff, 2017). En este sentido, la cantidad y calidad de las publicaciones en revistas de alto impacto se han convertido en uno de los principales indicadores para evaluar la productividad científica de las universidades (Gralka et al., 2019).

\section{Método}

La presente investigación, cuyo objetivo es analizar características del profesorado con perfil investigador en las universidades colombianas, es de tipo cuantitativo, no experimental.

La población de referencia está conformada por 4142 grupos de investigación reconocidos por el Sistema Nacional de Ciencia, Tecnología e Innovación adscritos a 82 universidades colombianas, clasificados en diferentes categorías A1, A, B, C y reconocidos por el Ministerio de Ciencia, Tecnología e Innovación. Esta información fue tomada del Sistema de Información GrupLac, dispuesta para visibilidad su existencia y productividad de los grupos de investigación avalados por distintas entidades públicas y privadas del país, cuya referencia son los resultados del último proceso de evaluación y medición generados en diciembre de 2017 (Colciencias, 2017).

Sobre esta población, se seleccionaron los conglomerados de grupos de investigación categorizados en A1 y A, pues se consideró que esta clasificación es otorgada a aquellas instancias que se caracterizan por una alta producción científica en revistas de alto impacto y por llevar a cabo procesos de formación de nuevo personal investigador en programas académicos de doctorado (Colciencias, 2017). Teniendo en cuenta lo anterior, se identificaron 1203 grupos de investigación adscritos a setenta universidades colombianas en los dos conglomerados seleccionados. Mediante un muestro probabilístico, de tipo aleatorio simple, se determinó una muestra representativa de 379 grupos de investigación (95\% de confianza y $5 \%$ de margen de error).

La recolección de información se llevó a cabo durante los meses de abril y mayo de 2018 y consistió en la aplicación de un cuestionario autosuministrado al profesorado líder de los grupos de investigación ${ }^{4}$. El cuestionario fue enviado a 1167 correos electrónicos asociados a profesorado líder de grupos de investigación. Se recibieron 350 respuestas completas (29.99\%); 182 respuestas incompletas (15.59\%); 64 declinaron (5.4\%) y no se recibió respuesta 571 participantes (48.92\%). Las respuestas completas pertenecen a profesorado vinculado en 48 de las 82 universidades colombianas.

\footnotetext{
${ }^{4}$ Antes de iniciar la aplicación del cuestionario se previó una opción para que el profesorado, mediante consentimiento informado, autorizara el proceso de tratamiento de datos. Adicionalmente, se trató de un cuestionario anonimizado.
} 
http://doi.org/10.15359/ree.24-3.17

El cuestionario fue diseñado a partir de la información obtenida en la revisión teórica previa. Se analizaron los siguientes factores: 1) características sociodemográficas (nivel de formación, ubicación geográfica y área de conocimiento de su último nivel de formación, edad, sexo, actividades científicas que desarrolla y tipo de vinculación laboral; 2) condiciones institucionales (carácter de la universidad, recursos financieros para actividades de investigación, recursos técnicos, infraestructura física para actividades de investigación, incentivos económicos por productividad científica, libertad para desarrollar actividades de investigación y asignación de tiempo en plan de trabajo para investigar; y finalmente 3) factores individuales (concepción de la investigación, colaboración, tipo de producción científica que genera, gestión externa de recursos para proyectos de investigación y número de proyectos de investigación desarrollados en su trayectoria).

Se utilizaron preguntas dicotómicas y escalas de medición de frecuencia de cinco opciones de respuesta ( 0 . Nada; 1 . Rara vez, 2 . Algunas veces, 3 . Muchísimas veces y 4 . Siempre), considerando que esta clasificación brinda dos compontes básicos al momento de la medición: la direccionalidad de la actitud del individuo, por un lado, y la intensidad en la que percibe el fenómeno (Cañadas Osinski y Sánchez Bruno, 1998).

Se llevó a cabo un análisis factorial exploratorio (AFE). La prueba de esfericidad de Bartlett con valores $(p<0.05)$ y el índice $\mathrm{KMO}$, con valores superiores a 0.60 , aconsejan la utilización de este tipo de técnicas (Ferrando y Lorenzo-Seya, 2014). Adicionalmente, la fiabilidad de los factores fue aceptable con valores que oscilan entre $a=0.718$ y $a=0.793$. Los resultados para los constructos sometidos a este análisis se muestran en la Tabla 1.

Tabla 1: Resumen análisis factorial exploratorio de variables

\begin{tabular}{llcccc}
\hline \multirow{2}{*}{ Constructo } & \multicolumn{1}{c}{ Dimensiones } & $\begin{array}{c}\text { Número de } \\
\text { variables }\end{array}$ & $\begin{array}{c}\text { Medida } \\
\text { KMO }\end{array}$ & $\begin{array}{c}\text { Varianza } \\
\text { explicada }\end{array}$ & $\begin{array}{c}\text { Alfa de } \\
\text { Cronbach }\end{array}$ \\
\hline \multirow{2}{*}{ Concepción de la investigación } & Producto & 3 & 0.654 & $60.50 \%$ & 0.723 \\
\cline { 2 - 6 } & Procesos & 3 & 0.600 & $64.44 \%$ & 0.718 \\
\hline Colaboración & Colaboración & 4 & 0.721 & $59.85 \%$ & 0.793 \\
\hline \multirow{2}{*}{ Tipo de producción científica } & Artículos de alto impacto & 4 & 0.712 & $80.12 \%$ & 0.763 \\
\cline { 2 - 6 } & Libro resultado de investigación & 3 & 0.682 & $64.33 \%$ & 0.720 \\
\hline
\end{tabular}

Nota: Elaboración propia. 
http://doi.org/10.15359/ree.24-3.17

http://www.una.ac.cr/educare

educare@una.ac.cr

Las 70 universidades colombianas de las que se obtuvo información, se divieron en tres grupos: a) crecimiento exponencial temprano (CET); b) crecimiento exponencial tardío (CETA); y c) crecimiento lineal e irregular (CLI), tomando como referencia la clasificación propuesta por Bucheli et al. (2012). En su estudio, analizaron el tipo de curva de crecimiento de la producción científica de las universidades entre 1958 y 2008 en revistas de alto impacto indexadas en Web of Science. Considerando la aceleración en el incremento de la producción científica de las universidades colombianas, sugierieron la siguiente clasificación de universidades (Bucheli et al., 2012):

- Crecimiento exponencial temprano. La curva de publicaciones ajusta como función exponencial. Además, las universidades han estado publicando investigaciones durante los últimos veinticinco años y han publicado más de 100 artículos por año durante los últimos dos años.

- Crecimiento exponencial tardío. La curva de publicaciones ajusta como función exponencial. Además, las universidades han estado publicando investigaciones en un período menor a 25 años y en los dos últimos años han publicado menos de 100 artículos por año.

- Crecimiento linear e irregular. La curva de publicaciones ajusta con una función lineal o la curva de publicaciones muestra una fluctuación fuerte en el número de publicaciones, habiendo algunos años en que no hay ninguna publicación.

Esta clasificación de referencia de universidades colombianas se actualizó para efectos del presente estudio al período 2001 y 2017. Para las 70, las universidades colombianas reportan producción científica en ese período de tiempo, según referencia de las colecciones Science Citation Index Expanded (SCI-EXPANDED); Social Sciences Citation Index (SSCl); Arts \& Humanities Citation Index (A\&HCl) y Emerging Source Citation Index (ESCl de Web of Science).

Para realizar la búsqueda, se utilizaron las etiquetas de campo OG (Organizaciones Nombre preferido) y CU (País). Se consideraron solo los artículos publicados en las diferentes colecciones y no hubo un criterio de idioma para filtrar la información. Adicionalmente, se limitó la búsqueda al período 2001-2017 y solo se consideraron aquellas publicaciones con primera autoría de profesorado vinculado a instituciones de educación superior reconocidas como universidades colombianas. El resultado arrojó 14.253 publicación de las universidades colombianas durante el período 2001 a 2017 (véase Tabla 2). 
http://doi.org/10.15359/ree.24-3.17

Tabla 2: Clasificación de universidades según productividad científica 2001 - 2017

\begin{tabular}{lccc}
\hline \multicolumn{1}{c}{ Clasificación } & $\begin{array}{c}\text { Total de universidades } \\
\text { con producción científica } \\
2001-2017\end{array}$ & $\begin{array}{c}\text { Porcentaje de } \\
\text { universidades con } \\
\text { respuesta de profesorado }\end{array}$ & $\begin{array}{c}\text { Porcentaje de profesorado } \\
\text { con perfil investigador que } \\
\text { respondieron el cuestionario } \\
\text { (muestra) }\end{array}$ \\
\hline $\begin{array}{l}\text { Crecimiento exponencial } \\
\text { temprano (CET) }\end{array}$ & 6 & $100 \%(6)$ & $55,5 \%(197)$ \\
$\begin{array}{l}\text { Crecimiento exponencial } \\
\text { tardío (CETA) }\end{array}$ & 17 & $64.7 \%(11)$ & $21,4 \%(76)$ \\
$\begin{array}{l}\text { Crecimiento lineal e } \\
\text { irregular (CLI) }\end{array}$ & 47 & $59,5 \%(28)$ & $21,7 \%(77)$ \\
\hline
\end{tabular}

Nota: Elaboración propia.

En cuanto al análisis de datos, se sometieron las características sociodemográficas, las condiciones institucionales y los factores individuales a prueba de comparación de medias para estudiar la existencia o no de diferencias respecto a los tres grupos de universidades clasificados, para lo cual se utilizó el estadístico $H$ Kruskal-Wallis. Finalmente, se compararon aquellas muestras que presentaron diferencias significativas $p<0.05$ con mediante un estudio de diferencias intergrupales con el uso del estadístico $U$ de Mann-Whitney. Es importante mencionar que la utilización de pruebas no paramétricas obececió a que las distribuciones muestrales no cumplieron con el supuesto de normalidad después de ser sometidas a las pruebas Shapiro-Wilk y Kolmogórov-Smirnov $(p<0.05)$.

\section{Resultados}

El profesorado con perfil investigador de las universidades colombianas tiene una edad media de 49 años. El 62.6\% son hombres y el $37.4 \%$ son mujeres. El $85.7 \%$ tienen título de doctorado y el $14.3 \%$ de maestría. La distribución del profesorado, según el área de formación disciplinar es: $23.4 \%$ ciencias naturales, $24.9 \%$ ingeniería y tecnología, el $14.0 \%$ a las ciencias médicas y de la salud, el $3.7 \%$ ciencias agrícolas, el $27.7 \%$ a las ciencias sociales y el $6.3 \%$ a las humanidades. Finalmente, el $29.4 \%$ del profesorado que conforma la muestra adelantó sus estudios avanzados en Europa, el $9.4 \%$ en Norteamérica, el 35.1\% en Suramérica y el $23.4 \%$ en Colombia. En la Tabla 3, se presentan los datos desagregados según la clasificación de crecimiento de la productividad científica en las universidades. 
http://doi.org/10.15359/ree.24-3.17

http://www.una.ac.cr/educare

educare@una.ac.cr

Tabla 3: Análisis descriptivo de variables sociodemográficas del profesorado

\begin{tabular}{|c|c|c|c|}
\hline Factores & CET & CETA & $\mathrm{CLI}$ \\
\hline \multicolumn{4}{|c|}{ Características generales del profesorado } \\
\hline \multicolumn{4}{|l|}{ Nivel de formación (\%) } \\
\hline Doctorado & $93.4 \%$ & $84.2 \%$ & $67.5 \%$ \\
\hline Maestría & $6.6 \%$ & $15.8 \%$ & $32.5 \%$ \\
\hline \multicolumn{4}{|c|}{ Ubicación geográfica (último grado) (\%) } \\
\hline Europa & $33.5 \%$ & $30.3 \%$ & $18.2 \%$ \\
\hline Norteamérica & $10.7 \%$ & $10.5 \%$ & $5.2 \%$ \\
\hline Centroamérica & $1 \%$ & $0 \%$ & $0 \%$ \\
\hline Suramérica & $32.5 \%$ & $39.5 \%$ & $37.7 \%$ \\
\hline Oceanía & $0,9 \%$ & $0 \%$ & $0 \%$ \\
\hline Colombia & $20.8 \%$ & $19.7 \%$ & $33.8 \%$ \\
\hline \multicolumn{4}{|c|}{ Área de conocimiento (\%) } \\
\hline Ciencias naturales & $32.2 \%$ & $9.2 \%$ & $15.6 \%$ \\
\hline Ingeniería y tecnología & $24.9 \%$ & $32.9 \%$ & $16.9 \%$ \\
\hline Ciencias médicas y de la salud & $13.2 \%$ & $15.8 \%$ & $14.3 \%$ \\
\hline Ciencias agrícolas & $5.6 \%$ & $0 \%$ & $2.6 \%$ \\
\hline Ciencias sociales & $19.3 \%$ & $34.2 \%$ & $42.9 \%$ \\
\hline Humanidades & $5.1 \%$ & $7.9 \%$ & $7.8 \%$ \\
\hline Edad (m) & $5 \%$ & $48 \%$ & $48 \%$ \\
\hline \multicolumn{4}{|c|}{ Sexo (\%) } \\
\hline Hombre & $61.4 \%$ & $69.7 \%$ & $58.4 \%$ \\
\hline Mujer & $38.6 \%$ & $30.3 \%$ & $41.6 \%$ \\
\hline \multicolumn{4}{|c|}{ Tipo de vinculación (\%) } \\
\hline No exclusividad & $38.6 \%$ & $43.4 \%$ & $57.1 \%$ \\
\hline Exclusividad & $61.4 \%$ & $56.6 \%$ & $42.9 \%$ \\
\hline
\end{tabular}

Nota: Elaboración propia. 
http://doi.org/10.15359/ree.24-3.17

El análisis de comparación de medias entre las características sociodemográficas del profesorado evidenció que su nivel de formación, el área disciplinar de su formación, la universidad en la que finalizó los estudios y el tipo de vinculación contractual con la universidad son variables que presentan un grado de significación $(p<0.05)$ de que corrobora la existencia de diferencias entre los tres grupos de universidades (véase Tabla 4).

Tabla 4: Características del profesorado y crecimiento de la productividad científica

\begin{tabular}{lc}
\hline Características del profesorado & $x^{2}(2)$ \\
\hline Nivel de formación & $30.34^{*}$ \\
Universidad en que culminó sus estudios & $8.77^{*}$ \\
Área de conocimiento & $21.76^{*}$ \\
Sexo & 2.332 \\
Tipo de vinculación contractual & $7.735^{*}$ \\
\hline${ }^{*} p<0.001$ &
\end{tabular}

Nota: Elaboración propia.

El nivel de formación del profesorado con perfil investigador en las universidades presenta diferencia significativa inter-grupos, según la prueba $H$ Kruskal-Wallis. La diferencia se explica debido a que en las universidades que presentan un crecimiento exponencial, temprano y tardío, tienen un mayor porcentaje de profesorado con doctorado que desarrolla actividades de investigación, como se evidenció en la Tabla 3, que las universidades con crecimiento líneal e irregular en las que predomina profesorado con maestría.

Respecto al área de conocimiento en los que desarrollan investigación el profesorado, aquellas universidades que tienen un crecimiento exponencial temprano se caracterizan por tener un mayor número de profesorado con perfil investigador en áreas de las ciencias naturales (32.2\%) frente a universidades con crecimiento tardío o lineal e irregular (9.2\% y $15.6 \%$ respectivamente). La segunda causa de la diferencia inter-grupos se explica en que las universidades con crecimiento exponencial tardío y las de crecimiento lineal e irregulal tienen un mayor número de docentes con perfil investigador en el área de las ciencias sociales (34.2\% y 42.9\%). Las otras áreas disciplinares no presentan un aporte causal a la existencia de diferencias entre el área de conocimiento y el crecimiento de la productividad científica de las universidades $(p>0.05)$.

Asimismo, el lugar de obtención del último grado académico por parte de profesorado también presenta diferencias significativas entre los grupos de universidades. Si bien entre aquellas que muestran un crecimiento exponencial temprano y tardío no existen diferencias 
http://doi.org/10.15359/ree.24-3.17

http://www.una.ac.cr/educare

educare@una.ac.cr

$(p>0.05)$, el profesorado de estos dos grupos de universidades se diferencia del vinculado a universidades con crecimiento lineal e irregular, tal como se evidencia en la Tabla 3 en la que se visualiza que las universidades con crecimiento exponencial se caracterizan por tener un mayor número de docentes formados en Europa y Norteamérica.

Finalmente, la otra diferencia encontrada está asociada a que existe una mayor proporción de profesorado con vinculación exclusiva en las universidades con crecimiento exponencial temprano (61.4\%) que en aquellas con crecimiento exponencial tardío y crecimiento lineal e irregular ( $56.6 \%$ y $42 . \%$ respectivamente). Sin embargo, se confirma que no existe diferencia significativa entre las universidades con crecimiento exponencial tardío y las de crecimiento lineal e irregular en relación con el tipo de vinculación de su profesorado $(p>0.05)$.

En el análisis de las condiciones institucionales, mediante prueba $H$ Kruskal-Wallis, se encontró que la existencia de incentivos económicos (salariales o bonificaciones extrasalariales) por producción científica, así como la libertad que se da al profesorado para escoger temas de investigación de su interés presentaron diferencias significativas entre los grupos de universidades $(p<0.001)$ (véase Tabla 5).

Tabla 5: Prueba Kruskal-Wallis para las condiciones institucionales

\begin{tabular}{lcccc}
\hline $\begin{array}{l}\text { Condiciones institucionales para desarrollar } \\
\text { actividad de investigación }\end{array}$ & $\overline{\mathrm{X}}(\sigma)$ & $\overline{\mathrm{X}}(\sigma)$ & $\overline{\mathrm{X}}(\sigma)$ & $X^{2}(2)$ \\
\hline Recursos financieros & $2.37(0.782)$ & $2.36(0.890)$ & $2.69(0.936)$ & 7.683 \\
$\begin{array}{l}\text { Oportunidad de vincular estudiantes de } \\
\text { pregrado y posgrado }\end{array}$ & $2.76(1.055)$ & $2.76(1.153)$ & $3.22(0.982)$ & 11.778 \\
Infraestructura física & $2.90(1.020)$ & $2.97(1.006)$ & $2.68(1.175)$ & 1.231 \\
Recursos técnicos & $2.83(0.988)$ & $3.11(0.858)$ & $2.65(1.121)$ & \multicolumn{1}{c}{6.791} \\
Incentivos económicos & $3.21(1.081)$ & $2.79(1.099)$ & $2.18(1.528)$ & $31.990^{*}$ \\
Estímulos no-económicos (viajes, sabáticos) & $2.55(0.992)$ & $2.37(0.936)$ & $2.39(1.114)$ & 2.211 \\
Ambiente de trabajo con colegas competentes & $3.02(0.906)$ & $2.84(0.925)$ & $3.08(0.900)$ & 3.120 \\
Libertad para escoger temas de investigación & $3.82(0.456)$ & $3.49(0.663)$ & $3.62(0.629)$ & $24.627^{*}$ \\
\hline
\end{tabular}

${ }^{*} p<0.001$

Nota: Elaboración propia. 
http://doi.org/10.15359/ree.24-3.17 http://www.una.ac.cr/educare educare@una.ac.cr

Para ahondar en los resultados, se estudiaron las diferencias inter-grupos utilizando el estadístico U Mann-Whitney. En el análisis, se halló que el otorgar incentivos económicos al profesorado por producción científica genera diferencia entre los tres grupos de universidades. Se presentó una diferencia con mayor significación entre las universidades con crecimiento exponencial temprano de su productividad científica y aquellas con crecimiento lineal e irregular $(p<0.001)$. En cuanto a las universidades con crecimiento exponencial, temprano y tardío, la diferencia tuvo un grado de significación menor $(p<0.05)$. Teniendo como referencia los datos descriptivos, el resultado indica que el profesorado vinculado a universidades con crecimiento exponencial temprano percibe con mayor intensidad (muchas veces) incentivos económicos por productividad científica $\bar{X}_{C E T}=3,21$, que aquel vinculado a las universidades que se clasifican en los otros tipos de crecimiento, el cual recibe incentivos algunas veces $\bar{X}_{C E T A}=2.79$ y $\bar{X}_{C L I}=2.18$.

Respecto a la libertad para escoger temas de investigación, en general el profesorado es libre para desarrollar actividades científicas en su campo disciplinar $\overline{\mathrm{X}}_{C E T}=3.82 ; \overline{\mathrm{X}}_{\text {CETA }}=3.49$ y $\overline{\mathrm{X}}_{C L I}=3.62$. Se encontró que es una característica que si bien genera diferencias significativas entre las universidades con crecimiento exponencial temprano y los otros dos grupos de clasificación de universidades $(p<0.001)$, no existe diferencia significativa entre el profesorado vinculado a universidades con crecimiento exponencial tardío y crecimiento lineal e irregular $(p>0.05)$.

Los factores individuales analizados en la Tabla 6, presentaron diferencias significativas en la concepción de la investigación que tiene el profesorado y el tipo de producción científica que derivan de sus proyectos de investigación.

Tabla 6 : Prueba Kruskal-Wallis para factores individuales

\begin{tabular}{lcccc}
\hline $\begin{array}{l}\text { Factores individuales para desarrollar } \\
\text { actividad de investigación }\end{array}$ & $\overline{\mathrm{X}}(\sigma)$ & $\overline{\mathrm{X}}(\sigma)$ & $\overline{\mathrm{X}}(\sigma)$ & $X^{2}(2)$ \\
\hline Concepción de la investigación (productos) & $2.82(0.791)$ & $2.64(0.709)$ & $2.86(0.774)$ & 4.923 \\
Concepción de la investigación (procesos) & $2.23(0.965)$ & $2.15(0.873)$ & $2.67(0.889)$ & $14.370^{*}$ \\
Colaboración & $2.40(0.649)$ & $2.16(0.656)$ & $2.27(0.761)$ & 2.942 \\
Trabajo individual & $0.94(0.927)$ & $1.08(1.017)$ & $1.21(1.05)$ & 3.557 \\
Producción científica (artículos de alto impacto) & $2.49(0.932)$ & $2.15(0.905)$ & $2.03(0.975)$ & $16.581^{*}$ \\
Producción científica (libros de investigación) & $1.40(0.843)$ & $1.61(0.805)$ & $1.99(0.864)$ & $25.480^{*}$ \\
\hline
\end{tabular}

${ }^{*} p<0.001$

Nota: Elaboración propia. 
http://doi.org/10.15359/ree.24-3.17

http://www.una.ac.cr/educare

educare@una.ac.cr

Al analizar el factor concepción de la investigación, se encontró que, en general, el profesorado concibe la investigación como producto con una mayor intensidad, en promedio, de $(\bar{X}=2.77)$ que aquellos grupos que la conciben como proceso ( $\bar{X}=2.35)$.

En el análisis inter-grupos, utilizando nuevamente la prueba U Mann-Whitney, las dimensiones de concepción de la investigación como producto y como proceso no presentaron diferencias significativas entre el profesorado vinculado a universidades que muestran un crecimiento exponencial de su productividad científica $(p=0.071)$ y $(p=0.438)$, respectivamente). Sin embargo, sí se encontraron diferencias significativas en la concepción de investigación del profesorado vinculado a universidades con crecimiento exponencial (temprano y tardío) frente a aquel profesorado que trabaja en universidades cuya productividad científica crece de manera lineal e irregular $(p=0.001)$. En las universidades con crecimiento exponencial, el profesorado concibe con menor intensidad la investigación como proceso $\left(\bar{X}_{C E T}=2.23\right.$ y $\left.\bar{X}_{\text {CETA }}=2.15\right)$ y como producto $\left(\bar{X}_{C E T}=2.82\right.$ y $\left.\bar{X}_{C E T A}=2.64\right)$, que en aquellas con crecimiento lineal e irregular $\left(\bar{X}_{C L I}=\right.$ 2.67 y $\left.\bar{X}_{C L I}=2.86\right)$, respectivamente).

El tipo de producción científica es otro factor que presenta diferencias importantes entre los grupos de universidades $(p<0.05)$, respecto a las universidades con crecimiento exponencial tardío y $(p<0.001)$ a las universidades con crecimiento lineal e irregular. Partiendo de este hallazgo, el profesorado vinculado a universidades con un crecimiento exponencial temprano genera con mayor intensidad $\left(\bar{X}_{C E T}=2.49\right)$ producción de artículos como resultado de investigación que el profesorado vinculado a los otros dos grupos de universidades $\left(\bar{X}_{\text {CETA }}=2.19\right.$ y $\left(\bar{X}_{C L I}=2.16\right)$.

Por otro lado, existe diferencias significativas en la producción científica de libros resultado de investigación $(p<0.05)$ entre los tres grupos de universidades. El profesorado vinculado a universidades con crecimiento lineal e irregular producen con mayor intensidad $\left(\bar{X}_{C L I}=2.10\right)$ libros como resultado de investigación que el profesorado vinculado a los otros dos grupos de universidades $\left(\bar{X}_{\text {CETA }}=2.15\right.$ y $\left.\bar{X}_{C L I}=2.03\right)$.

\section{Discusión y conclusiones}

El análisis de las características del profesorado con perfil investigador vinculado a universidades colombianas (i.e., condiciones sociodemográficas, condiciones institucionales y factores individuales) ha permitido identificar algunas diferencias en función del tipo del grado de productividad científica de la universidad a la que se vincula (i.e., crecimiento exponencial temprano, crecimiento exponencial tardío, crecimiento exponencial linear e irregular).

Una de las variables que muestra diferencias entre el profesorado es el área disciplinar de formación. Para el caso colombiano, el hecho de que las universidades con un crecimiento exponencial temprano tengan un mayor número de docentes con perfil investigador en áreas 
http://doi.org/10.15359/ree.24-3.17

de las ciencias naturales, mientras que en los otros dos grupos de universidades predomine el profesorado que investiga en áreas de las ciencias sociales explican parcialmente esa diferencia. Edgar y Geare (2013) indicaron que ese crecimiento en las ciencias básicas se debe a que los tipos y procesos de investigación, en algunas de sus disciplinas, facilitan la obtención de resultados de investigación que puedan ser sometidos a publicación de manera más rápida que en las áreas humanísticas. Además, en las ciencias sociales, las artes o las humanidades, los tiempos de obtención de resultados que puedan ser publicados suelen ser mayores como lo evidenciaron Edgar y Geare (2013). Esta situación supone un reto interesante para la evaluación de la producción científica en las universidades colombianas, si se considera que un alto porcentaje del profesorado participa de actividades de investigación, como se mostró en los resultados, se ha formado en el área de las ciencias sociales.

La dedicación exclusiva del profesorado al desarrollo de actividades de investigación en una universidad específica es otra característica que se relaciona con el crecimiento exponencial temprano de su productividad científica. Bazeley (2010) encontró que el profesorado que tiene el compromiso y la persistencia para orientar su trabajo al desarrollo de procesos de investigación (task orientation), sumado a la oportunidad de vincularse a una universidad que le brinde condiciones satisfactorias para dedicarse de manera exclusiva a este tipo de actividades (opportunity and resources) maximizan la productividad científica institucional. Albert et al. (2018) reafirman la importancia de la satisfacción laboral del profesorado en el incremento de su productividad científica.

Resulta relevante poner este asunto en consideración, dado que en Colombia es común identificar profesorado trabajando de manera simultánea en varias universidades, con diferentes tipos de contratación, motivado por la posibilidad de obtener mayor retribución económica. La no exclusividad, como lo habían afirmado Méndez Sayago y Vera Azaf (2015), puede limitar su orientación específica a desarrollar actividades de investigación que impacten en el crecimiento de la productividad científica de las universidades, hecho que se evidencia en esta investigación, pues este el tipo de vinculación mayoritario en profesorado perteneciente a universidades con crecimiento exponencial tardío o lineal.

Una de las condiciones institucionales que se ha establecido para fomentar la producción científica ha sido otorgar incentivos económicos por publicación al profesorado en las universidades. En Colombia, el Decreto 1279 de 1992, por el cual se establece el régimen salarial y prestacional del personal docente de las Universidades Estatales, en el artículo 6 reconoce la productividad académica como factor para asignar puntos salariales al profesorado y el artículo 10 contempla los diferentes tipos de productos que otorgan incremento salarial. Méndez Sayago y Vera Azaf (2015) encontraron que el incentivo otorgado en universidades públicas tiene un efecto positivo sobre la productividad científica del profesorado, hecho que se corrobora en esta investigación. Para igualar las condiciones que recibe el profesorado en universidades públicas, 
http://doi.org/10.15359/ree.24-3.17

http://www.una.ac.cr/educare

educare@una.ac.cr

algunas universidades privadas han promovido políticas de incentivos por productividad científica (bonificaciones anuales); sin embargo, los mismos autores consultados estimaron que estas no tienen el mismo efecto que en las universidades públicas, pues, al no considerarse el incentivo como un factor constitutivo de salario, su incidencia en el largo plazo es menor, hecho que se corrobora en esta investigación. En esta misma línea se sitúan los resultados aportados por otras investigaciones previas, en las que se indica que los incentivos proporcionados a las universidades o directamente al profesorado (ej., incremento salarial, reducción de las horas de docencia) no implican necesariamente un incremento de su productividad (ej., Carrington et al., 2018; Cerasoli et al., 2014; Jørgensen y Hanssen, 2018).

La libertad para escoger temas de investigación está relacionada de manera positiva con la productividad científica. Edgar y Geare (2013), así como Kwiek (2018), encontraron que la autonomía que se brinda al profesorado para desarrollar investigación científica en sus campos de interés es una práctica gerencial, y parte de la cultura organizacional, que ha generado un impacto muy significativo en la productividad científica de las universidades, dada la posibilidad que tiene el profesorado para profundizar en sus áreas de formación y desplegar sus redes de colaboración nacional e internacional. En las universidades colombianas, sin diferencia alguna en el crecimiento de la productividad científica, se reconoce, de manera favorable, esta práctica que se funda en que el reconocimiento institucional, de las capacidades individuales del profesorado, incide de manera positiva en su motivación, variable que se ha relacionado con una alta productividad científica.

Los resultados obtenidos para las dos dimensiones de concepción de la investigación indican que estas no son mutuamente excluyentes; hecho que, aunque ya había sido corroborado por Stubb et al. (2014) en su investigación con estudiantes doctorales, aún no había sido analizado en profesorado universitario. Los resultados expuestos permiten inferir que estas dos dimensiones de lo que se concibe como investigación se sobreponen en el profesorado universitario en universidades colombianas. Es decir, que al interior de las universidades existen docentes que conciben la investigación como un proceso de indagación, desarrollado en diferentes etapas (revisión teórica, diseño de instrumentos, sistematización de información), sin dejar de lado que la obtención de resultados como productos (publicaciones) con alto nivel de visibilidad e impacto derivados de cada proyecto genera motivación individual (incentivos o reputación). Este último es un hecho propio de la cultura moderna de la academia, como lo afirmaron Stubb et al. (2014), que ha llevado al profesorado a realizar investigación con la finalidad de publicar muchos artículos en un corto período de tiempo y que ha abierto el debate académico sobre la pertinencia de los sistemas de evaluación de la ciencia, que pueden favorecer la cantidad y no la calidad de la producción científica como lo han afirmado Abramo y D'Angelo (2014). 
Frente a estas dos dimensiones de la concepción de la investigación, la evidencia encontrada en este trabajo permite realizar las siguientes consideraciones: 1) Respecto a la concepción de la investigación como proceso, en Colombia, las universidades con crecimiento lineal e irregular de su productividad científica tienen un mayor porcentaje de profesorado con perfil investigador con nivel de formación en maestría. En este nivel de formación es posible que cualquier actividad de investigación que desarrolle o se conciba como una etapa de crecimiento y transformación para consolidarse como personal investigador, lo que coincide con los resultados encontrados por Stubb et al. (2014) en su investigación sobre concepción de la investigación en estudiantes de doctorado. 2) En cuanto a la concepción de la investigación como producto, existen condiciones institucionales como la existencia de incentivos y la falta de oportunidades para vincularse de manera exclusiva a una universidad, que pueden ser aprovechadas por el profesorado para sacar beneficio personal, dada la necesidad que algunas universidades tienen de incrementar rápidamente su productividad científica como ocurre en otros contextos (Altbach y Wit, 2019).

En resumen, al analizar diferentes características del profesorado con perfil investigador que está vinculado a universidades colombianas, se encontró que, al considerar los tipos de crecimiento de la productividad científica de las universidades a las que se encuentra vinculado, hay diferencias entre el profesorado. En este sentido, el estudio aporta evidencia empírica, tanto en las condiciones institucionales (estructura organizacional para la investigación y prácticas de gestión de la investigación) como en factores individuales, lo cual explica la existencia de las diferencias en el desarrollo y consolidación de las actividades de investigación en las universidades colombianas.

El estudio abre la posibilidad a que, en futuras investigaciones, se analicen los efectos que la gestión de la investigación en las universidades genera sobre su productividad científica, con el objetivo de ampliar el conocimiento sobre la forma como se conciben y se desarrollan las actividades científicas al interior de las instituciones de educación superior en Colombia. Asimismo, se convierte en un insumo para continuar desarrollando investigación, en el campo de la gestión educativa, que favorezca la implementación de buenas prácticas en los procesos de dirección de este tipo de organizaciones.

\section{Declaración de Material complementario}

Este artículo tiene disponible como material complementario:

- La versión preprint del artículo en https://doi.org/10.5281/zenodo.3366357 
http://doi.org/10.15359/ree.24-3.17

http://www.una.ac.cr/educare

educare@una.ac.cr

\section{Referencias}

Abramo, G. y D'Angelo, C. A. (2014). How do you define and measure research productivity? Scientometrics, 101(2), 1129-1144. https://doi.org/10.1007/s11192-014-1269-8

Abramo, G., D'Angelo, C. A. y Soldatenkova, A. (2017). An investigation on the skewness patterns and fractal nature of research productivity distributions at field and discipline level. Journal of Informetrics, 11(1), 324-335. https://doi.org/10.1016/j.joi.2017.02.001

Albert, C., Davia, M. A. y Legazpe, N. (2018). Job satisfaction amongst academics: The role of research productivity. Studies in Higher Education, 43(8), 1362-1377. https://doi.org/10.10 $\underline{80 / 03075079.2016 .1255937}$

Altbach, P. G. y de Wit, H. (2019). Too much academic research is being published. International Higher Education, 96, 2-3. https://doi.org/10.6017/ihe.2019.96.10767

Amara, N., Landry, R. y Halilem, N. (2015). What can university administrators do to increase the publication and citation scores of their faculty members? Scientometrics, 103(2), 489-530. https://doi.org/10.1007/s11192-015-1537-2

Bazeley, P. (2010). Conceptualising research performance. Studies in Higher Education, 35(8), 889903. https://doi.org/10.1080/03075070903348404

Bornmann, L. y Leydesdorff, L. (2017). Skewness of citation impact data and covariates of citation distributions: A large-scale empirical analysis based on Web of Science data. Journal of Informetrics, 11(1), 164-175. https://doi.org/10.1016/j.joi.2016.12.001

Brew, A. (2001). Conceptions of research: A phenomenographic study. Studies in Higher Education, 26(3), 271-285. https://doi.org/10.1080/03075070120076255

Brew, A., Boud, D., Namgung, S. U., Lucas, L. y Crawford, K. (2016). Research productivity and academics' conceptions of research. Higher education, 71(5), 681-697. https://doi. org/10.1007/s10734-015-9930-6

Bucheli, V., Díaz, A., Calderón, J. P., Lemoine, P., Valdivia, J. A., Villaveces, J. L. y Zarama, R. (2012). Growth of scientific production in Colombian universities: An intellectual capital-based approach. Scientometrics, 91(2), 369-382. https://doi.org/10.1007/s11192-012-0627-7

Cañadas Osinski, I. y Sánchez Bruno, A. (1998). Categorías de respuesta en escalas tipo likert. Psicothema, 10(3), 623-631. http://www.psicothema.com/psicothema.asp?id=191

Carrington, R., O'Donnell, C., \& Prasada Rao, D. S. (2018). Australian university productivity growth and public funding revisited. Studies in Higher Education, 43(8), 1417-1438. https:// doi.org/10.1080/03075079.2016.1259306 
Cattaneo, M., Meoli, M. y Signori, A. (2016). Performance-based funding and university research productivity: The moderating effect of university legitimacy. Journal Technology Transfer, 41(1), 85-104. https://doi.org/10.1007/s10961-014-9379-2

Cerasoli, C. P., Nicklin, J. M. y Ford, M. T. (2014). Intrinsic motivation and extrinsic incentives jointly predict performance: A 40-year meta-analysis. Psychological Bulletin, 140(4), 9801008. https://doi.org/10.1037/a0035661

Colciencias. (2017). Modelo de medición de grupos de investigación, desarrollo tecnológico o de innovación y de reconocmiento de investigadores del sistema nacional de ciencia, tecnología e innovación, año 2017. Departamento Administrativo de Ciencia, Tecnología e Innovación, Colciencias.

Daraio, C. (2019). Econometric approaches to the measurement of research productivity. En W. Glänzel, H. F. Moed, U., Schmoch y M. Thelwall (Eds.), Springer Handbook of Science and Technology Indicator (pp. 633-666). Springer. https://doi.org/10.1007/978-3-030-02511-3 24

Delgado Hurtado, C., Rubiano Ovalle, O., Rengifo Rodas, C. F., y Rojas Pineda, E. (2017). Research capacities of universities: Estimation of parameters and modeling of the dynamics of the research systems. Biotecnología en el Sector Agropecuario y Agroindustrial, 15(2), 121-133. http://dx.doi.org/10.18684/BSAA(15)121-133

Edgar, F. y Geare, A. (2013). Factors influencing university research performance. Studies in Higher Education, 38(5), 774-792. https://doi.org/10.1080/03075079.2011.601811

Ferrando, P. J. y Lorenzo-Seva, U. (2014). El análisis factorial exploratorio de los ítems: Algunas consideraciones adicionales. Anales de Psicologia, 30(3), 1170-1175. https://doi. org/10.6018/analesps.30.3.199991

Frenken, K., Heimeriks, G. J. y Hoekman, J. (2017). What drives university research performance? An analysis using the CWTS Leiden Ranking data. Journal of Informetrics, 11(3), 859-872. https://doi.org/10.1016/j.joi.2017.06.006

Gralka, S., Wohlrabe, K. y Bornmann, L. (2019). How to measure research efficiency in higher education? Research grants vs. publication output. Journal of Higher Education Policy and Management, 41(3), 322-341. https://doi.org/10.1080/1360080X.2019.1588492

Ion, G. y Castro-Ceacero, D. (2017). Transitions in the manifestations of the research culture of Spanish universities. Higher Education Research \& Development, 36(2), 311-324. https://doi. org/10.1080/07294360.2016.1208153

Jørgensen, F. y Hanssen, T. E. S. (2018). Research incentives and research output. Higher Education: The International Journal of Higher Education Research, 76(6), 1029-1049. https:// doi.org/10.1007/s10734-018-0238-1 
http://doi.org/10.15359/ree.24-3.17

http://www.una.ac.cr/educare

educare@una.ac.cr

Kyvik, S. y Aksnes, D. W. (2015). Explaining the increase in publication productivity among academic staff: a generational perspective. Studies in Higher Education, 40(8), 1438-1453. https://doi.org/10.1080/03075079.2015.1060711

Kwiek, M. (2018). Academic top earners. Research productivity, prestige generation, and salary patterns in European universities. Science and Public Policy, 45(1), 1-13. https://doi. org/10.1093/scipol/scx020

Lucio, J., Guevara, A., Perea, G. I., Torralba Barreto, D. R., Romero, I. C., Ramírez, D., Castellanos, J. C., Mora Holguín, H., Sánchez, E. C., Castro, N., García, J. M., Caho, D. M., Nupia, C. M., Cardona Mora, L. y Pardo Martínez, C. I. (2017). Indicadores de ciencia y tecnología. Colombia 2016. OcyT. Observatorio Colombiano de Ciencia y Tecnología.

Macfarlane, B. (2017). The paradox of collaboration: A moral continuum. Higher Education Research \& Development, 36(3), 472-485. https://doi.org/10.1080/07294360.2017.1288707

Méndez Sayago, J. y Vera Azaf, L. (2015). Salarios, incentivos y producción intelectual docente en la universidad pública en Colombia. Apuntes del CENES, 34(60), 95-130. https://doi. org/10.19053/22565779.3281

Nguyen, H. T. L. y Meek, V. L. (2016). Key problems in organizing and structuring university research in Vietnam: The lack of an effective research "behaviour formalization" system. Minerva, 54(1), 45-73. https://doi.org/10.1007/s11024-016-9289-6

Ospina Bozzi, M. L., Bozzi Ángel, P., Ahumada Barona, J. y Otero Ruíz, E. (2016). Colciencias, 30 años: Memorias de un compromiso. Colciencias.

Plata, J. J. (2013). Colciencias cuarenta años. Aprendizajes organizacionales y retos en las sociedades del conocimiento (Cap. 2). En M. Salazar (Ed.), Colciencias cuarenta años. Entre la legitimidad, la normatividad y la práctica (pp. 62-117). Observatorio Colombiano de Ciencia y Tecnología. https://www.ocyt.org.co/proyectos-y-productos/colcienciascuarenta-anos-entre-la-legitimidad-la-normatividad-y-la-practica/

Stubb, J., Pyhältö, K. y Lonka, K. (2014). Conceptions of research:The doctoral student experience in three domains. Studies in Higher Education, 39(2), 251-264. https://doi.org/10.1080/0307 $\underline{5079.2011 .651449}$

Upadhyaya, P. y Rajasekharan Pillai, K. (2019). Management research outcome: a comparative assessment of BRICS nations. Studies in Higher Education, 44(9), 1567-1578. https://doi.org $\not 10.1080 / 03075079.2018 .1455083$ 